\title{
Erratum to: Fourth cranial nerve: surgical anatomy in the subtemporal transtentorial approach and in the pretemporal combined inter-intradural approach through the fronto-temporo-orbito-zygomatic craniotomy. A cadaveric study
}

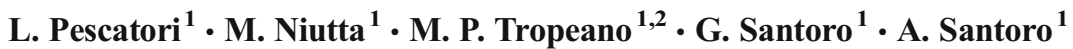

Published online: 3 October 2016

(C) Springer-Verlag Berlin Heidelberg 2016

Erratum to: Neurosurgical Review

10.1007/s10143-016-0777-9

In the manuscript the Authors reported two illustrative cases: the first is the case of a 15-years old boy who underwent subtemporal transtentorial approach for the surgical removal of a cavernoma localized at pons and mesecephalon, the second is the case of a 20-years old girl who underwent a combined pre- temporal intra and extra dural approach with FTOZ craniotomy for a lesion localized in the Meckel's cave. There' $\mathrm{s}$ mistake in the order of figures: the figures 5-6-7 refer to the second illustrative case, while the figure 8-9-10 refer to the first illustrative case.

The online version of the original article can be found at http://dx.doi. org/10.1007/s10143-016-0777-9.

M. P. Tropeano

mariapia.tropeano@libero.it

1 Department of Neurology and Psichiatry-Neurosurgery, "La

Sapienza", University of Rome, Viale del Policlinico 155,

Rome, Italy

2 Via Francesco Manfra n³, 83100 Avellino, Italy 